\title{
Alterações morfofuncionais decorrentes de dois treinamentos de força distintos: treinamento de força com pesos e o treinamento de força no meio líquido Morphofunctional alterations resulting from two different strength training: strength training with weights and strength training in water
}

Rodrigo Pereira*, Dilmar P. Guedes Junior, M.Sc.**, Fabrício Madureira***

*Mestrando em Ciências da Saúde pela Universidade Federal de São Paulo (Unifesp/Baixada Santista), Docente na Faculdade de Educação Física de Santos (Fefis/Unimes) - Docente na Faculdade da Praia Grande (FPG), **Docente na Faculdade de Educação Física de Santos (Fefis/Unimes), Docente na Faculdade de Educação Física e Esportes (FEFESP/UNISANTA) - Membro do Centro de Estudos em Fisiologia do Exercício e Treinamento (CEFIT), ${ }^{* * *}$ Doutorando em Comportamento Motor pela Universidade de São Paulo (USP) - Docente na Faculdade de Educação Física de Santos(Fefis/Unimes)

\section{Resumo}

O objetivo do presente estudo foi comparar as modificaçóes morfofuncionais decorrentes de dois protocolos de treinamentos de força em ambientes distintos: musculação e water force. 31 indivíduos foram divididos em dois grupos, MUSC e WF. Ambos os grupos treinaram durante seis meses, seis vezes por semana, e realizaram testes no início e final do programa, para avaliar a força muscular máxima dinâmica. Os testes realizados foram: supino reto (1 RM); um minuto de flexôes de braço (RES_BR); um minuto de abdominais (RES_ABD); maior número de saltos sobre uma corda durante um minuto (RES_MMII). Para avaliação da composiçáo corporal foi utilizado o protocolo de Pollock. Foi calculada a estimativa da secção transversa de braço (ASTM_BR) e coxa (ASTM_CX).
O grupo MUSC obteve os seguintes aumentos significativos $(\mathrm{P}<0,01)$ : 1RM $(17,48 \%)$; RES_ABD (25,1\%); RES_BR (40,94\%); RES_MMII (28,34\%); ASTM_BR (16,17\%); ASTM_CX (16,63\%). O WF obteve as seguintes melhoras significativas $(\mathrm{P}<0,01)$ : 1RM (27,88\%); RES_ABD (30,87\%); RES_BR $(40,69 \%)$; RES_MMII $(46,15 \%)$; ASTM_BR $(14,04 \%)$; ASTM_CX $(11,63 \%)$. Entretanto, não foram observadas interaçóes estatísticas significativas entre os grupos, exceto para massa corporal ( $\mathrm{P}=$ 0,0106). Os dois tipos de treinamentos MUSC e WF se demonstraram eficientes em relação às modificações morfofuncionais, força de membros superiores, hipertrofia muscular e resistência de força.

Palavras-chave: treinamento, musculação, hidroginástica, força. 


\section{Abstract}

The aim of this study was to compare morphofunctional modifications resulting from two protocols of strength training in different environments: weight training and water force. 31 subjects were divided into two groups WF and MUSC. Both groups have trained for six months, six times a week. Tests were conducted, at the beginning and at the end of the program, to assess the maximum dynamic muscle strength. Tests included: bench press (1 RM); one minute of push-ups (RES_BR); one minute of abdominal (RES_ABD); greater number of jumps on a rope for one minute (RES_MMII). For body composition assessment protocol was used Pollock. The estimate of the transverse section of arm (ASTM_BR) and thigh (ASTM_CX) was calculated. The MUSC group has achieved the following significant increases $(\mathrm{P}<0.01)$ : $1 \mathrm{RM}$ (17.48\%); RES_ABD (25.1\%); RES_BR (40.94\%); RES_MMII (28.34\%); ASTM_BR (16.17\%); ASTM_ CX (16.63\%). WF obtained the following significant improvement $(\mathrm{P}<0.01)$ : $1 \mathrm{RM}(27.88 \%)$; RES_ABD (30.87\%); RES_BR (40.69\%); RES_MMII (46.15\%); ASTM_BR (14.04\%); ASTM_CX (11.63\%). However, no statistically significant interactions between groups, except for body mass $(P=0.0106)$ were observed. The two types of training MUSC and WF demonstrated to be efficient in relation to morpho-functional modifications, upper limb strength, muscular hypertrophy and strength endurance.

Key-words: training, aquatic training, weight training, force.

\section{Introdução}

Programas de treinamento resistido têm sido investigados intensamente nos últimos anos possibilitando maior conhecimento sobre ordem de exercícios, variaçôes de repetições, séries e velocidades de execução [1-3]. Ainda, dados robustos apontam para os potenciais efeitos deste modelo de treinamento nos diferentes esportes [4-8], em seres humanos em condiçóes especiais $[9,10]$ e nas diferentes fases da vida [11-13]. Entretanto, uma grande quantidade de indivíduos tem lotado academias de ginástica no Brasil, na procura deste modelo de treino para potencializar a estética, através do aumento da massa muscular e redução da massa gorda, correlação esta que é evidenciada em diferentes achados na literatura acadêmica [14-17].

Como o desenvolvimento da força é uma manifestação adaptativa do sistema neuromuscular frente à sobrecarga, pode-se esperar que o treinamento de força utilizando a resistência da água também seja uma alternativa para o aumento de força e hipertrofia. Contudo, são recentes os estudos que demonstram os efeitos de protocolos de treinamento de força que utilizam as propriedades da água e equipamentos específicos para este ambiente como forma de sobrecarga. Trabalhos como os de Borreani et al. [18] realizados com indivíduos jovens; Robinson et al. [19] com jogadores de vôlei; Rocha et al. [20] indivíduos universitários; Rodrigues et al. [21] atletas de futsal; Bartolotto et al. [22] adolescentes; Moreira et al. [23] mulheres pós-menopausa; Bergamin et al. [24] idosos; Fernandez-Lao et al. [25] portadores de câncer, apontam para a eficiência da potencialização da força em ambiente aquático. Entretanto, é intrigante refletir sobre duas questôes, que são: 1) O potencial do treinamento de força na água é equivalente ao mesmo modelo de treinamento desenvolvido em ambiente terrestre?; 2) Se o aumento da força detectado nos trabalhos supracitados resultantes de intervençóes aquáticas, pode influenciar na composiçâo corporal dos participantes de treinamento resistido neste ambiente?

\section{Objetivo}

Comparar as modificaçóes morfofuncionais decorrentes de dois protocolos de treinamento de força em ambientes distintos: treinamento de força com pesos e treinamento de força no meio líquido.

\section{Material e métodos}

O estudo foi submetido ao comitê de ética da Universidade Metropolitana de Santos. Os participantes assinaram o termo de consentimento 
livre e esclarecido, detalhando a qual tipo de experimento eles seriam submetidos e, posteriormente, foram submetidos a exame médico e avaliaçôes físicas propostas pelo estudo.

Foram avaliados 31 indivíduos divididos em dois grupos, MUSC e WF. O grupo MUSC com 14 indivíduos (seis mulheres e oito homens), média de idade de $24,7(4,27)$ anos e que realizaram o treinamento de força com pesos, e o grupo WF com 17 indivíduos (oito mulheres e nove homens), média de idade de 22,8(3,69) anos e que realizaram o treinamento de força no meio líquido. Ambos os grupos treinaram 6 vezes por semana por um período de 24 semanas, e realizaram um pré-teste (semana 2 ) e um pós-teste (após a semana 24).

\section{Testes de força}

Para avaliar a força muscular máxima foi utilizado o teste de 1 repetição máxima(1RM) no supino reto descrito por Kraemer [26]. A resistência muscular de membros superiores foi avaliada pelo teste de 1 minuto de flexóes de braço (RES_BR) [27] a resistência muscular dos músculos flexores do tronco foi medida através do teste de 1 minuto de abdominais (RES_ABD) [27] e resistência de membros inferiores (RES_MMII) [27] que foi avaliada contando o número total de saltos sobre uma corda durante 1 minuto, com a mesma estando a 60 centímetros do chão.

\section{Avaliaçáo da composiçáo corporal}

Os testes de avaliaçóes antropométricas foram os de circunferência de braço (CCF_BR), antebraço (CCF_ATB), cintura (CCF_CT), abdome (CCF_ABD), coxa (CCF_CX) e perna (CCF_PN), seguindo o protocolo de Jackson e Pollock [28]. Para testar a potência de membros superiores foi utilizado o arremesso de Medicine Ball de 2kg (POT_MMSS) Pollock [29] e de membro inferior que foi o salto horizontal (POT_MMII). Para a composição corporal foi mensurada a massa corporal total (MC), a dobra cutânea de tríceps (DC_TRI), bíceps (DC_BI), peito (DC_PEI), abdome (DC_ABD), coxa (DC_CX), perna medial (DC_PM) e perna lateral (DC_PL). Para estimar a área de secção transversa do braço (AST_BR) e área de secção transversa da coxa (AST_CX) foram utilizados dois cálculos proposto por Malina \& Katzmarzyk [30].

Foi aplicada a prova estatística de Shapiro-Wilk, bem como a inspeção do gráfico de quantis (qq-plot) com o objetivo de verificar se as observaçôes seguiam uma distribuição próxima à distribuição normal. Uma vez que as variáveis avaliadas apresentavam distribuição que podia ser aproximada pela distribuição normal, optou-se por descrevê-las utilizando à média, como estimador de posição e o desvio padrão, como estimador de dispersão. Empregou-se o intervalo de confiança, com coeficiente de confiança definido em $95 \%$, como estimador da incerteza relativa à alteração média calculada em função do treinamento. Para verificar o efeito do treinamento e comparar se os métodos de treino diferiam entre si, empregou-se um modelo de efeitos mistos, considerando como efeito fixo o período do treino (pré ou pós-treino), o método de treino (WF ou MUSC) e a interação entre os fatores período e método de treino. Foram modelados como efeitos aleatórios o intercepto e os coeficientes angulares obtidos para os termos período e método de treino, considerando a influência dos voluntários sobre tais termos. Aceitou-se a significância estatística em $\alpha \leq 0,05$.

\section{Resultados}

\section{Características antropométricas da amostra}

Tabela I - Descrição da massa corporal e da soma das dobras cutâneas de acordo com o periodo e método de treino.

\begin{tabular}{ccccc}
\hline & \multicolumn{2}{c}{$\begin{array}{c}\text { Massa corporal } \\
(\mathrm{kg})\end{array}$} & \multicolumn{2}{c}{$\begin{array}{c}\text { Soma dobras } \\
\text { cutâneas }\end{array}$} \\
& Pré & Pós & Pré & Pós \\
\hline \multirow{3}{*}{ MUSC } & 66,97 & 68,70 & 50,93 & 48,29 \\
& $(11,27)$ & $(11,33)$ & $(23,51)$ & $(21,05)^{*}$ \\
& 66,29 & 65,96 & 54,62 & 52,50 \\
WF & $(10,60)$ & $(11,28) \propto$ & $(26,57)$ & $(26,89)^{*}$ \\
\hline
\end{tabular}

Os dados são apresentados na forma de média (desvio padrão). *Interação estatisticamente significante ( $P=$ 0,0106), entre período e ambiente. aIndica diferença estatisticamente significante $(P=0,0402)$ em relação ao pré treino. 
Conforme indicando na tabela I, houve uma interação estatisticamente significante entre ambiente e período de treino, de tal maneira que no grupo (MUSC ambiente 1), observou-se um aumento médio de $1,73 \mathrm{~kg}$ na massa corporal, por sua vez, no grupo (WF ambiente 2) a massa corporal apresentou uma redução média de $0,76 \mathrm{~kg}$; indicando que o comportamento da massa corporal total em função do treino pode depender do tipo de treino empregado. Os resultados demonstraram que o programa do grupo WF obteve melhores modificações na massa corporal total. A soma de dobras cutâneas apresentou uma redução média de $2,3 \mathrm{~mm}$ em função do treinamento, caracterizando diferenças estatisticamente significativas nas condições experimentais para os diferentes momentos, mas tal alteração foi similar entre ambos os métodos de treino.

Tabela II - Descrição da circunferência de braço e antebraço de acordo com o periodo e método de treino.

\begin{tabular}{lllrl}
\hline & \multicolumn{2}{c}{ Braço $(\mathrm{cm})$} & \multicolumn{2}{c}{ Antebraço $(\mathrm{cm})$} \\
\cline { 2 - 5 } & Pré & \multicolumn{1}{c}{ Pós } & Pré & \multicolumn{1}{c}{ Pós } \\
\hline \multirow{2}{*}{ MUSC } & 28,48 & 30,19 & 24,93 & 26,24 \\
& $(2,94)$ & $(2,87)^{\propto}$ & $(2,01)$ & $(2,24)^{\propto}$ \\
\multirow{2}{*}{ WF } & 28,74 & 30,38 & 24,92 & 25,73 \\
& $(3,09)$ & $(3,86)^{\propto}$ & $(2,35)$ & $(2,77)^{\propto}$
\end{tabular}

Os dados são apresentados na forma de média (desvio padrão). andica diferença estatisticamente significante ( $P$ $\leq 0,0001$ ) em relação ao pré treino.

De acordo com os dados na tabela II, ambos os programas apresentaram efeitos estatisticamente significativos para as condiçóes de intervenção nos momentos pré e pós-teste. A circunferência do braço apresentou um aumento médio de 1,67 $\mathrm{cm}$; que foi similar entre os dois protocolos. Por sua vez na circunferência do antebraço o aumento foi de aproximadamente $0,9 \mathrm{~cm}$, e também não se detectou diferença estatística entre os grupos.
Ao se avaliar o efeito de ambos os programas de treinamento sobre as circunferência relacionada com o acúmulo de gordura central, não se observou nenhuma alteração significativa na circunferência abdominal. Tanto para a circunferência de coxa, quanto para a circunferência de perna, foram respectivamente observados aumentos significativos de $2,1 \mathrm{e}$ $0,9 \mathrm{~cm}$ em média, identificando-se diferenças estatísticas nos momentos pré e pós-teste, em ambas as condições experimentais, entretanto, não foi detectada diferença entre os grupos.

Tabela IV - Descrição da área de secção transversa de braço e coxa de acordo com o periodo e método de treino.

\begin{tabular}{ccccc}
\hline & \multicolumn{2}{c}{$\begin{array}{c}\text { ASTM\# do braço } \\
\left(\mathbf{c m}^{2}\right)\end{array}$} & \multicolumn{2}{c}{$\begin{array}{c}\text { ASTM\# da coxa } \\
\left(\mathbf{c m}^{2}\right)\end{array}$} \\
\cline { 2 - 5 } & Pré & Pós & Pré & Pós \\
\hline \multirow{2}{*}{ MUSC } & 49,71 & 57,75 & 136,18 & 158,83 \\
& $(15,88)$ & $(16,95)^{\natural}$ & $(32,80)$ & $(42,28)^{\natural}$ \\
WF & 51,34 & 58,55 & 140,64 & 157,00 \\
& $(17,09)$ & $(22,18)^{\natural}$ & $(42,97)$ & $(45,46)^{\natural}$ \\
\hline
\end{tabular}

\# área de secção transversa muscular. Os dados são apresentados na forma de média (desvio padrão). $\not$ Indica diferença estatisticamente significante $(P \leq 0,001)$ em relação ao pré treino.

A área de secçáo transversa apresentou aumentos significativos estatisticamente tanto para membros superiores quanto em membros inferiores apenas nos diferentes momentos das intervençôes. Nos braços observou-se aumento médio de $7,6 \mathrm{~cm}^{2}$; por sua vez, na coxa tal aumento foi de $19,56 \mathrm{~cm}^{2}$.

Características de força, resistência de força e potência muscular.

Tabela III - Descrição da circunferência de abdome, coxa e perna de acordo com o período e método de treino.

\begin{tabular}{|c|c|c|c|c|c|c|}
\hline & \multicolumn{2}{|c|}{ Abdome $(\mathrm{cm})$} & \multicolumn{2}{|c|}{ Coxa $(\mathrm{cm})$} & \multicolumn{2}{|c|}{ Perna $(\mathrm{cm})$} \\
\hline & Pré & Pós & Pré & Pós & Pré & Pós \\
\hline MUSC & $79,31(6,61)$ & $79,15(6,94)$ & $52,02(3,39)$ & $54,61(4,23)^{x}$ & $34,99(2,23)$ & $35,93(2,01)^{\alpha}$ \\
\hline WF & $79,18(7,63)$ & $79,28(7,47)$ & $53,79(3,94)$ & $54,61(4,23)$. & $36,12(2,70)$ & $36,96(2,86) d$ \\
\hline
\end{tabular}

Os dados são apresentados na forma de média (desvio padrão). $\alpha$ Indica diferença estatisticamente significante $(P \leq$ 
Tabela V - Descrição da carga máxima dinâmica de supino e potência de membros superiores e inferiores de acordo com o periodo e método de treino.

\begin{tabular}{lcccccc}
\hline & \multicolumn{2}{c}{ Carga máxima no supino $(\mathrm{kg})$} & \multicolumn{2}{c}{ Potência M.S. $(\mathrm{m})$} & \multicolumn{2}{c}{ Potência de $M . I(\mathrm{~m})$} \\
\hline Pré & Pós & Pré & Pós & Pré & Pós \\
\hline MUSC & $53,43(18,17)$ & $62,77(20,95)^{*}$ & $4,99(1,42)$ & $5,31(1,21)^{\propto}$ & $2,24(0,33)$ & $2,41(0,33)^{\alpha}$ \\
WF & $52,75(24,72$ & $67,45(24,69)^{*}$ & $4,54(1,49)$ & $5,22(1,52)^{\propto}$ & $2,19(0,39)$ & $2,52(0,32)^{\alpha}$ \\
\hline
\end{tabular}

Os dados são apresentados na forma de média (desvio padrão).; ${ }^{*}$ Indica diferença estatisticamente significante $(P=$

$0,0001)$ em relação ao pré treino; Indica diferença estatisticamente significante $(P \leq 0,0001)$ em relação ao pré treino.

Tabela VI - Descrição da resistência de membros superiores, abdominal e membros inferiores de acordo com o periodo e método de treino.

\begin{tabular}{lcccccc}
\hline & \multicolumn{2}{c}{ Resistência M.S. (rep.) } & \multicolumn{2}{c}{ Resistência ABD (rep.) } & \multicolumn{2}{c}{ Resistência M.I. (rep.) } \\
\hline & Pré & Pós & Pré & Pós & Pré & Pós \\
\hline MUSC & $29,31(8,89)$ & $41,31(8,04)^{\propto}$ & $54,54(17,05)$ & $68,23(12,81) \propto$ & $28,62(12,54)$ & $36,73(9,48)^{*}$ \\
WF & $31,53(10,78)$ & $44,36(9,45)^{\propto}$ & $48,19(15,87)$ & $68,23(12,81) \propto$ & $27,43(13,07)$ & $40,09(18,89)^{*}$ \\
\hline
\end{tabular}

Os dados são apresentados na forma de média (desvio padrão); $\not$ Indica diferença estatisticamente significante ( $P \leq$

$0,0001)$ em relação ao pré treino; * Indica diferença estatisticamente significante $(P=0,0003)$ em relação ao pré treino.

Independentemente do treino empregado, foi observado um aumento médio de $6,4 \mathrm{~kg}$ na carga máxima do supino após o período de treinamento, caracterizando diferenças estatisticamente significantes apenas entre os momentos de intervenção. A potência gerada tanto por membros superiores, quanto inferiores apresentou aumento estatisticamente significativo após o período de treinamento, independente do protocolo empregado. Tais aumentos foram de 0,53 e $0,22 \mathrm{~m}$; para membros superiores e inferiores, respectivamente.

Considerando, ainda, os efeitos de ambos os programas de treinamento no membro superior, evidenciou-se um aumento significativo médio de 12,4 repetiçóes na resistência muscular que ocorreu de maneira independente ao tipo de treino empregado. A resistência abdominal apresentou um aumento significativo estatisticamente, apenas para os momentos de intervenção nos dois programas de aproximadamente 13,9 repetiçóes. O mesmo comportamento foi observado na resistência de membros inferiores, de tal forma, que ao final do período de treino encontrou-se um aumento médio de 8,5 repetiçóes nos grupos, não se detectando diferenças entre os mesmos.

\section{Discussão}

Uma das questôes deste trabalho tinha como intuito avaliar se os efeitos potenciais do treina- mento de força na água seriam equivalentes ao mesmo modelo de treinamento desenvolvido em ambiente terrestre. Os dados encontrados permitem inferir que os grupos investigados sobre os efeitos do treinamento resistido em diferentes ambientes (MUSC e WF) apresentaram melhoras significativas nos momentos pré e pós-intervenção, mas não se detectou diferença entre os grupos. Especificamente para o grupamento muscular abdominal, Bressel et al. [31] demonstraram efeitos positivos do treinamento aquático para este grupamento muscular. Entretanto, em outro estudo, Bressel et al. [32] quando compararam a eletromiografia de diferentes exercícios abdominais realizados em ambiente terrestre e aquático, o ambiente terrestre apresentou resultados superiores. Os achados do presente trabalho demonstraram diferenças relativa entre pré e pós-treinamento de $32 \%$ para o grupo MUSC e $31,8 \%$ para o grupo WF corroborando o trabalho de Rocha et al. [33] que investigou a potência dos músculos abdominais em programas de treinamento de força em ambientes aquático e terrestre, com duração de 12 semanas, e verificaram ajustes de força significativos e similares em ambos os grupos terra e água.

No presente estudo, ambos os grupos evidenciaram uma diferença significativa para a variável força, ou seja, os dois programas de treinamento foram eficientes em relação à força 
e resistência de força para membros superiores, com maior destaque para o grupo WF que demonstrou uma diferença absoluta de $15 \mathrm{~kg}$ para o supino $(15,2 \%)$ e de 13 repetiçóes no teste de flexão de braço entre pré e pós-treinamento, apresentando uma diferença de $57,1 \%$. Os resultados corroboram o estudo realizado por Tsourlou et al. [34], o qual observou um aumento entre $25,7-29,4 \%$ na força muscular no teste de $3 \mathrm{RM}$ em idosos que realizaram um treinamento de força na água.

Com relação ao aumento da hipertrofia muscular, apesar de vários experimentos terem sido realizados investigando o potencial do ambiente aquático para a maximização da força utilizando-se diferentes materiais [18], em diferentes profundidades de imersão do corpo [35], o estudo de Poyhonen et al. [36] foi um dos primeiros a detectar magnitudes hipertróficas significativas para membros inferiores de mulheres jovens submetidas a treinamento de força em ambiente aquático. Já, Rodrigues et al. [21] confirmam o potencial hipertrófico do ambiente aquático também para membros inferiores de atletas de futsal, entretanto poucos trabalhos na literatura acadêmica apontam para modificações significativas da massa muscular de membros superiores. Neste trabalho os resultados indicam aumentos não apenas da potência de membros superiores e inferiores mas também para o aumento hipertrófico destas estruturas.

$\mathrm{Na}$ questão 2 deste trabalho, que se propunha investigar se os efeitos na composição corporal eram de magnitudes similares entre os treinos nos diferentes ambientes, os resultados corroboraram os achados de Greene et al. [37] que investigaram sujeitos com sobrepeso e obesos, bem como o trabalho de Colado et al. [38] que investigou mulheres pós-menopausa, todos submetidos a treino de força nos dois ambientes, portanto o aumento da hipertrofia muscular foi concomitante a diminuição da gordura corporal.

\section{Conclusão}

Os resultados do presente estudo sinalizam que o treinamento com pesos no meio líquido pode ser benéfico em relação às modificações morfofuncionais, principalmente a força de membros superiores, hipertrofia muscular, resistência de força dos testes aplicados, potência de membros superiores e inferiores e diminuição das dobras cutâneas. Não foram encontradas modificaçôes em relação à circunferência de abdome, embora tenha ocorrido aumento significativo na resistência deste mesmo grupamento muscular. $\mathrm{O}$ treinamento de força no meio líquido pode ser considerado como uma alternativa interessante para o treinamento dessa capacidade física, sendo este capaz de promover efeitos semelhantes aos exercícios com pesos fora da água. De acordo com os objetivos propostos neste estudo, os resultados sugerem que esse tipo de programa de exercícios pode ser útil para pessoas que possuem algum tipo de limitação para se exercitar em terra ou, ainda, aquelas pessoas que tem preferência pela prática de exercícios no meio líquido.

\section{Referências}

1. Simão R, Polito MD, Viveiros L, Farinatti PTV. Influência da manipulação na ordem dos exercícios de força em mulheres treinadas sobre o número de repetiçóes e percepção de esforço. Rev Bras Ativ Física Saúde 2012;7(2):53-61.

2. Walker $S$, Hakkinen K. Similar increases in strength after short-term resistance training due to different neuromuscular adaptations in young and older men. J Strength Cond Res 2014;21:21.

3. Carregaro RL, Gentil P, Brown LE, Pinto RS, Bottaro M. Effects of antagonist pre-load on knee extensor isokinetic muscle performance. J Sports Sci 2011;29(3):271-8.

4. Brito J, Vasconcellos F, Oliveira J, Krustrup P, Rebelo A. Short-term performance effects of three different low-volume strength-training programmes in college male soccer players. J Hum Kinet 2014;40:121-8.

5. Lawton TW, Cronin JB, McGuigan MR. Strength testing and training of rowers: a review. Sports Med 2011;41(5):413-32.

6. Girold S, Jalab C, Bernard O, Carette P, Kemoun G, Dugue B. Dry-land strength training vs. electrical stimulation in sprint swimming performance. J Strength Cond Res 2012;26(2):497-505.

7. Aguiar EJ, Morgan PJ, Collins CE, Plotnikoff RC, Callister R. Efficacy of interventions that 
include diet, aerobic and resistance training components for type 2 diabetes prevention: a systematic review with meta-analysis. Int J Behav Nutr Phys Act 2014;11(1):2.

8. Schranz N, Tomkinson G, Olds T. What is the effect of resistance training on the strength, body composition and psychosocial status of overweight and obese children and adolescents? A Systematic review and meta-analysis. Sports Med 2013;43(9):893-907.

9. Cheema BS, Chan D, Fahey P, Atlantis E. Effect of progressive resistance training on measures of skeletal muscle hypertrophy, muscular strength and health-related quality of life in patients with chronic kidney disease: a systematic review and meta-analysis. Sports Med 2014;44(8):1125-38.

10. Busch AJ, Webber SC, Richards RS, Bidonde J, Schachter CL, Schafer LA, et al. Resistance exercise training for fibromyalgia. Cochrane Database Syst Rev 2013;20(12).

11. Liu CJ, Latham NK. Progressive resistance strength training for improving physical function in older adults. Cochrane Database Syst Rev 2009;8(3).

12. Behringer M, Vom Heede A, Matthews M, Mester J. Effects of strength training on motor performance skills in children and adolescents: a meta-analysis. Pediatr Exerc Sci 2011;23(2):186-206.

13. Faigenbaum AD, Kraemer WJ, Blimkie CJ, Jeffreys I, Micheli LJ, Nitka M, et al. Youth resistance training: updated position statement paper from the national strength and conditioning association. J Strength Cond Res 2009;23(5 Suppl):60:79.

14. Davitt PM, Pellegrino JK, Schanzer JR, Tjionas $\mathrm{H}$, Arent SM. The effects of a combined resistance training and endurance exercise program in inactive college female subjects: does order matter? J Strength Cond Res 2014;28(7):1937-45.

15. Delshad M, Ghanbarian A, Mehrabi Y, Sarvghadi F, Ebrahim K. Effect of strength training and short-term detraining on muscle mass in women aged over 50 years old. Int J Prev Med 2013;4(12):1386-94.

16. Villanueva MG, He J, Schroeder ET. Periodized resistance training with and without supplementation improve body composition and performance in older men. Eur J Appl Physiol 2014;114(5):891-905.

17. Sillanpaa E, Hakkinen A, Nyman K, Mattila M, Cheng S, Karavirta L, et al. Body composition and fitness during strength and/or endurance training in older men. Med Sci Sports Exerc 2008;40(5):950-8.
18. Borreani S, Colado JC, Furio J, Martin F, Tella $\mathrm{V}$. Muscle activation in young men during a lower limb aquatic resistance exercise with different devices. Phys Sportsmed 2014;42(2):80-7.

19. Robinson LE, Devor ST, Merrick MA, Buckworth J. The effects of land vs. aquatic plyometrics on power, torque, velocity, and muscle soreness in women. J Strength Cond Res 2004;18(1):84-91.

20. Rocha AC, Vilarinho R, Gherardi F, Bulo F, Barboza M, Dubas JP, et al. Alteraçóes morfofuncionais causadas pelo treinamento de força no meio líquido. Fitness \& Performance Journal 2007;6(3):188-94.

21. Rodrigues H, Bonfim F, Gherardi F, Madureira F, Guedes Júnior D. Análise da resposta do treinamento resistido no meio líquido para membros inferiores de jogadores de futsal profissional. $30^{\circ}$ Encontro Nacional de Profissionais de Educação Física-ENAPEF 2004.

22. Bartolotto F, Ysis W, Florêncio R, Jr DPG, Madureira F. Efeito do treinamento aquático na composição corporal de adolescentes. Coleção Pesquisa em Educação Física 2011;10(5):73-8.

23. Moreira LDF, Fronza FCAO, Santos RN, Teixeira LR, Kruel LFM, Lazaretti-Castro M. High-intensity aquatic exercises (HydrOS) improve physical function and reduce falls among postmenopausal women. Menopause 2013;20(10):1012-9.

24. Bergamin M, Ermolao A, Tolomio S, Berton L, Sergi G, Zaccaria M. Water-versus land-based exercise in elderly subjects: effects on physical performance and body composition. Clin Interv Aging 2013 27;8:1109-17.

25. Fernández-Lao C, Cantarero-Villanueva I, Ariza-Garcia A, Courtney C, Fernández-de-las-Peñas C, Arroyo-Morales M. Water versus land-based multimodal exercise program effects on body composition in breast cancer survivors: a controlled clinical trial. Supportive Care in Cancer 2013;21(2):521-30.

26. Kraemer WJ, Ratamess NA. Fundamentals of resistance training: progression and exercise prescription. Med Sci Sports Exercise 2004;36(4):674-88.

27. Pollock ML, Wilmore JH. Exercícios na saúde e na doença. Avaliação e prescrição para prevenção e reabilitação. Rio de Janeiro: Medsi; 1993.

28. Jackson AS, Pollock ML. Generalized equations for predicting body density of men. Br J Nutr 1978;40(03):497-504.

29. Pollock ML, Wilmore JH, Fox SM. Exercise in health and disease: evaluation and pres- 
cription for prevention and rehabilitation. Philadelphia:WB Saunders Philadelphia; 1990.

30. Malina RM, Katzmarzyk PT. Physical activity and fitness in an international growth standard for preadolescent and adolescent children. Food Nutr Bull 2006;27(4):S295-313.

31. Bressel E, Dolny DG, Vandenberg C, Cronin JB. Trunk muscle activity during spine stabilization exercises performed in a pool. Phys Ther Sport 2012;13(2):67-72.

32. Bressel E, Dolny DG, Gibbons M. Trunk muscle activity during exercises performed on land and in water. Med Sci Sports Exercise 2011;43(10):1927-32.

33. Rocha A, Dubas J, Madureira F, Guedes Júnior $\mathrm{D}$, editors. Comparação do treinamento abdominal dentro e fora d'água. $19^{\circ}$ Congresso Internacional de Educação Física-FIEP; 2004.

34. Tsourlou T, Benik A, Dipla K, Zafeiridis A, Kellis S. The effects of a twenty-four-week aquatic training program on muscular strength performance in healthy elderly women. J Strength Cond Res 2006;20(4):811-8.

35. Colado JC, Borreani S, Pinto SS, Tella V, Martin F, Flandez J, et al. Neuromuscular responses during aquatic resistance exercise with different devices and depths. J Strength Cond Res 2013;27(12):3384-90.

36. Poyhonen T, Sipila S, Keskinen KL, Hautala A, Savolainen J, Malkia E. Effects of aquatic resistance training on neuromuscular performance in healthy women. Med Sci Sports Exerc 2002;34(12):2103-9.

37. Greene NP, Lambert BS, Greene ES, Carbuhn AF, Green JS, Crouse SF. Comparative efficacy of water and land treadmill training for overweight or obese adults. Med Sci Sports Exerc 2009;41(9):1808-15.

38. Colado JC, Triplett NT, Tella V, Saucedo P, Abellán J. Effects of aquatic resistance training on health and fitness in postmenopausal women. Eur J Appl Physiol 2009;106(1):113-22.

\section{Envie seu artigo!}

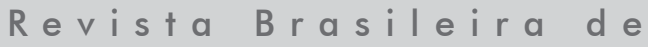
F IS I O LOG I A Do EXER C Í C I O Brazilian Journal of Exercise Physiology Órgão Oficial da Sociedade Brasileira de Fisiologia do Exercício

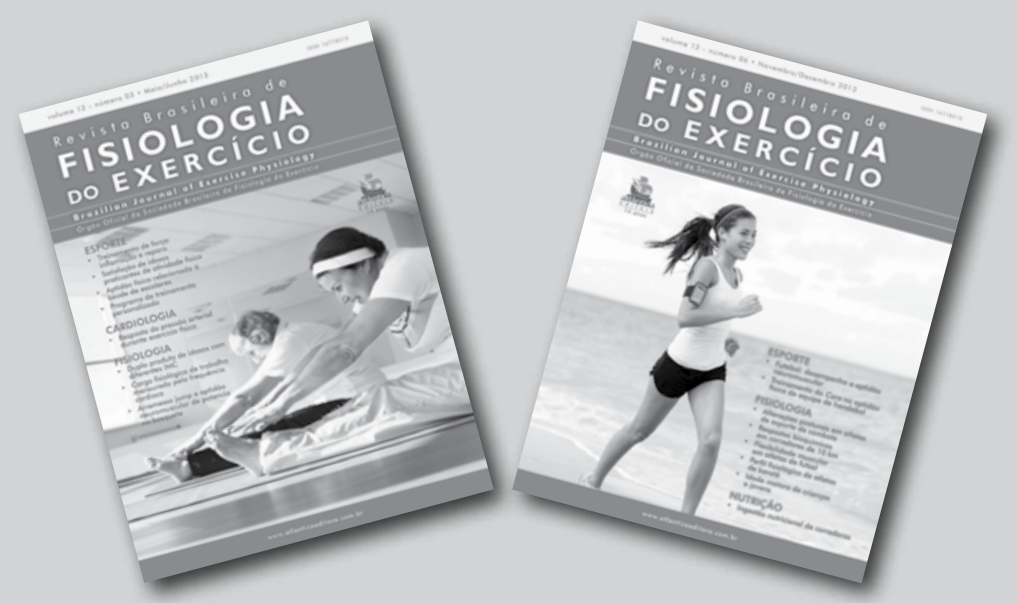

Tel: (11) 3361-5595 / artigos@atlanticaeditora.com.br 